University of Wollongong

Research Online

Faculty of Engineering and Information

Faculty of Engineering and Information

Sciences - Papers: Part B

Sciences

2019

\title{
Numerical and experimental studies on a new variable stiffness and damping magnetorheological fluid damper
}

Hui Huang

Fuzhou University

Shuaishuai Sun

University of Wollongong, ssun@uow.edu.au

Shumei Chen

Fuzhou University

Weihua Li

University of Wollongong, weihuali@uow.edu.au

Follow this and additional works at: https://ro.uow.edu.au/eispapers1

Part of the Engineering Commons, and the Science and Technology Studies Commons

Research Online is the open access institutional repository for the University of Wollongong. For further information contact the UOW Library: research-pubs@uow.edu.au 


\title{
Numerical and experimental studies on a new variable stiffness and damping magnetorheological fluid damper
}

\author{
Abstract \\ This article describes a new magnetorheological fluid damper; its damping and stiffness are variable and \\ controllable through the compact structure of two damping units and a spring. The ability of variable \\ stiffness and damping enables it to be used for more effective vibration control in wide-band excitation \\ wave occasions. First, the effective stiffness and output damping force are calculated in theory. Then, the \\ magnetic field is simulated by finite element analysis. After the prototype of the new magnetorheological \\ fluid damper is developed and machined, the damper's performances are tested in a hydraulic actuated \\ MTS machine, including stiffness variability and damping variability in different current. The successful \\ development, numerical calculation, and experimental testing shows that the new damper not only \\ outputs a controllable damping force but also occurs as the great variable stiffness in a certain range, \\ which makes the true design and implementation of the concept of variable stiffness and damping. \\ Disciplines \\ Engineering | Science and Technology Studies

\section{Publication Details} \\ Huang, H., Sun, S., Chen, S. \& Li, W. (2019). Numerical and experimental studies on a new variable \\ stiffness and damping magnetorheological fluid damper. Journal of Intelligent Material Systems and \\ Structures, 30 (11), 1639-1652.
}




\title{
Numerical and experimental studies on a new variable stiffness and damping magnetorheological fluid damper
}

\author{
Hui Huang ${ }^{1,3}$, Shuaishuai Sun², Shumei Chen ${ }^{1,3}$, Weihua $\mathbf{L i}^{2}$
}

\begin{abstract}
This paper describes a new magnetorheological (MR) fluid damper, its damping and stiffness are variable and controllable through the compact structure of two damping units and a spring. The ability of variable stiffness and damping enables it to be used for more effective vibration control in wide-band excitation wave occasions. Firstly, the effective stiffness, and output damping force are calculated in theory. Then the magnetic field is simulated by finite element analysis. After the prototype of the new MR fluid damper is developed and machined, the damper's performances are tested in a hydraulic actuated MTS machine, including stiffness variability and damping variability in different current. The successful development, numerical calculation, experiment testing shows that the new damper not only outputs a controllable damping force but also it occurs the great variable stiffness in a certain range, which make the true design and implementation of the concept of variable stiffness and damping.
\end{abstract}

Keyword

magnetorheological fluid; variable stiffness and damping; vibration control; numerical and experimental; structure design.

\section{Introduction}

Traditional way of vibration control is a passive control way, generally including vibration damping, vibration isolation, vibration absorbing, etc. The principle of vibration reduction is to absorb the energy of the main structure. The basic structure of vibration controlled device is generally composed of damping unit and elastic unit, which has been applied widely because of its simple structure and low cost. However, this way has its own limitations, such as its damping coefficient and elastic stiffness cannot be changed once the mechanism has been designed. When the natural frequency of controlled device is same or close to that of excitation vibration, the effect is the best. If the difference between them reaches a certain value, the effect of vibration control is not obvious or even gets worse (Talbot, 2004). Therefore, the concept and model of variable damping or stiffness are constantly proposed for vibration reduction. The variable damping can change the resonance magnitude of resonance by adjusting the dissipated energy, and the variable stiffness can change the transmissibility by changing the natural frequency of the controlled device, while the damping or stiffness can be varied controllability, in real-time and reversibility. Many researchers have come up with the basic idea of using a variable damping system to provide effective vibration control (Li et al., 2009; Sun et al., 2014). Worden et al. (1992) designed an oil and gas spring in single cylinder type as a shock absorber and analyzed its model. Kwang (1997) established the model of automotive monotube hydraulic dampers. All the above variable damping or

${ }^{1}$ School of Mechanical Engineering and Automation, Fuzhou University, China

${ }^{2}$ School of Mechanical, Materials and Mechatronics Engineering, University of Wollongong, Australia

${ }^{3}$ Key Laboratory of Fluid Power and Intelligent Electro-Hydraulic Control (Fuzhou University), Fujian Province University, China stiffness controlled device are very complicated.

To vary and control damping or stiffness, one of most effective vibration control ways is semi-active control, which combines the advantages of passive control and active control. It does not need to input big energy to the device as well as passive control, and its own characteristics can be varied to achieve the tracking for optimal response in real-time, which make the effect of vibration reduction be close to that of active control. Controlling the damping or stiffness in a semi-active control way has been an attractive research solution, such as semi-active frictional damper, magnetorheological fluid damper and adaptive tuned mass damper (Du et al., 2005; Gsell et al., 2007). Magnetorheological (MR) fluid dampers are typical semi-active dampers with variable damping capability that have been widely used for vibration control due to their reliability and fast response (Li et al., 2003). Choi et al. (2005) proposed an MR fluidbased semi-active isolator used to protect the avionics and sensitive instruments in aircraft and helicopters, and studied the performance of the isolator under the control of a skyhook controller. Spencer et al. (1998) used the MR damper to study seismic response reduction by carrying out a series of earthquake tests. ${ }^{1}$

In order to further improve the vibration reduction performance of vehicle suspensions, variable stiffness has also been tried. Youn and Hac (1995) developed a variable stiffness suspension by employing a semi-active air spring to vary the stiffness of suspension among three discrete values. The experimental result verified the effectiveness of variable stiffness on vibration control. However, compared with MR fluid technology, the air spring is

Shumei Chen, School of Mechanical Engineering and Automation, Fuzhou University, Fuzhou 350116, China.

Email: smchen@fzu.edu.cn

Weihua Li,School of Mechanical, Materials and Mechatronics Engineering, University of Wollongong, Northfields Avenue, Wollongong, NSW 2522, Australia

Email:weihuali@uow.edu.au 
complicated because the air pressure must be controlled by an air pump, which is complicated and costly. In order to vary stiffness by using MRF, Li et al. (2009) developed an MRF-based air spring which can vary its stiffness and damping by controlling the excitation current applied to an MR valve. A typical variable stiffness device for vibration reduction based on MR technology is the magnetorheological elastomer (MRE) isolator, which is usually used for protecting structures from earthquakes or huge wind (Zhang et al.,2008; Deng et al., 2006; Hitchcock et al., 2004; Li , 2013; Behrooz et al., 2014). Li et al. fabricated a and highly adjustable MRE isolator which increased the force by up to $1579 \%$ and the stiffness by up to $1730 \%$.

Based on the successful implementation of variable stiffness or damping devices, research directions have originated because of the appearance of the dual controlability of stiffness and damping (Liu et al., 2005A; Liu et al., 2008b). Sun et al. (2015) reported an isolator whose damping and stiffness can be simultaneously controlled by MR fluid and elastomer, in which MR fluids is used for varying damping and elastomer for varying stiffness. Raja et al. (2014) developed another variable stiffness and damping device: an MR fluid damper-liquid spring suspension system for heavy off-road vehicles. This device is capable of changing its damping as well as stiffness and its maximum force can reach to $10000 \mathrm{~N}$, but its stiffness and damping can't be controlled separately and its stroke is limited. Recently, Greiner-Petter et al. (2014) reported a unique structure employing MR fluid to realize the characteristics of variable stiffness and damping. Zhang et al. (2009) developed a variable stiffness and damping MR valve based on isolator. Its dynamic response showed that the stiffness could be adjusted in a relative large range. However, the structures above are complicated and not compact, impeding their practical applications.

In order to make the structure more compact, the function of variable stiffness and damping should be integrated into one device. Sun et al. (2015) developed a new MR fluid damper through the compact assembly of two MRF damping units and a spring. Experimental testing and modeling of this variable stiffness and damping MRF damper make the true design and implementation of the concept of variable stiffness and damping feasible. Sun et al. (2017) also developed a shock absorber for vehicle suspension with the characteristics of both variable damping and stiffness. Although these devices are relatively compact in structure, but only the inverse models of output force obtained by experiment and parameter identification are provided in their papers. Due to the limited working conditions of the experiment, not all the working states of these devices can be fully reflected. There is no positive model for variable stiffness, which makes them impossible to be controlled accurately in practical application. The reasons for the variation of stiffness and the relationship between stiffness and damping are not explained in theory in their papers.

In this paper, it develops a new compact MR fluid damper which adopts the integration of outer piston and inner cylinder based on the combination of inner and

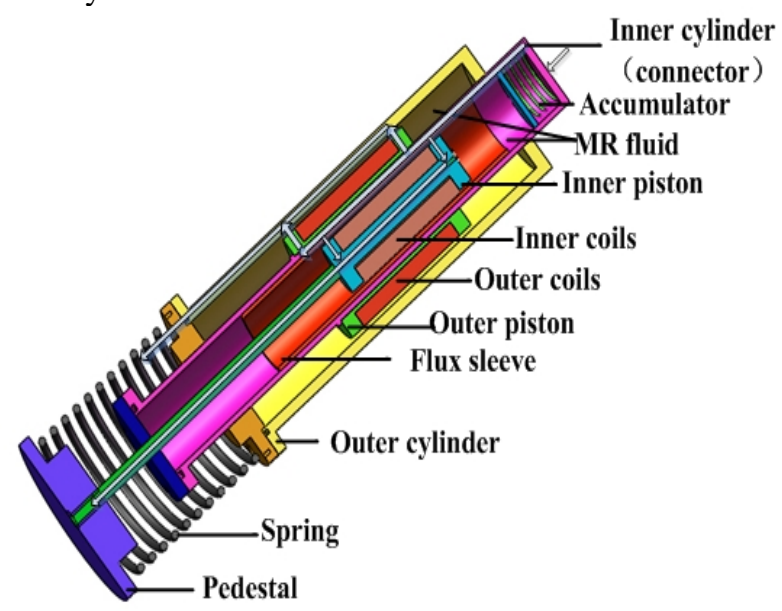

Figure 1. Structure of the new damper

outer damping units. Its variable damping and stiffness can make it is used in the vibrating occasions which have wide range of vibration frequency range. The variation of stiffness and damping can be analyzed and calculated in theory, so as to control it accurately. Firstly, the effective stiffness and output force are calculated in theory. Then the prototype is designed and machined. Finally, the experiment test is carried out, then the data between experiment and theoretical calculation are compared and analyzed.

\section{Design and model}

\subsection{Structure of MR fluid damper}

Figure 1 shows the structure of the new MR fluid damper which is mainly composed of an outer damping unit, an inner damping unit and a spring. The outer damping unit is mainly composed of inner cylinder, outer piston with excitation coil, outer cylinder and MF fluid. Then the outer unit is connected to a spring which is fixed on the pedestal in series form. The inner damping unit is mainly composed of flux sleeve mounted on the inner cylinder, a rod, inner piston with excitation coil and MF fluid. One end of the rod is connected to inner piston and the other is fixed on the pedestal, so the inner piston is not a moving piece. In the structure, the inner cylinder is as a connector to vibrating object. It can not only drive the outer piston to have relative movement with outer piston, but also drive the flux sleeve to have relative movement with the inner piston, so the inner and outer units are in parallel form. If the outer cylinder has movement which can compress the spring, and the spring starts to work and provide stiffness. The two excitation coils are powered by direct current $I_{1}$ and $I_{2}$ respectively, passing through magnetic fields. The inner damping force and the outer damping force are controlled by regulation of $I_{1}$ and $I_{2}$ respectively. By controlling magnetic fields on the MR fluid, the variable stiffness is implemented by the outer unit and spring, while the variable damping force is mainly implemented by the inner unit.

\subsection{Working principle}


Form the transmission path of force shown in Figure 1, the working model of the variable stiffness and damping damper can be demonstrated in Figure 2, where two different connection modes of this device are shown. The damping force of each unit is dependent on the crucial properties of MR fluid, such as the shear yield stress and zero-field viscosity, so a coulomb friction element $f_{1}$ in parallel with a viscous element $c_{1}$ is used to characterize the inner unit. Similarly, another coulomb friction element $f_{2}$ in parallel with a viscous element $c_{2}$, in series connection with a spring, is used to describe the outer unit. While the $f$ is controlled by the magnetic field which is generated by the applied current and it takes the main work of damping force. For the new damper, the damping variability is mainly controlled by the current $I_{1}$ applied to the inner unit. When the current $I_{1}$ is small, the damping force $f_{1}$ is relative small, however, it tends to be bigger as $I_{1}$ grows. The stiffness variability is controlled by the current $I_{2}$ applied to the outer unit. In this model, the shear yield force $f_{2}$ always should be bigger than the elastic force of spring when the applied current $I_{2}$ reaches a certain high point, and there is no relative slip between the outer cylinder and the inner cylinder, so the viscous element $c_{2}$ is blocked. In this case, the spring should be compressed with the inner cylinder when a external force $F_{i}(t)$ has a displacement, so the displacements of the outer and inner cylinder are the same. Otherwise, when the applied current $I_{2}$ is very small, there will be a relative motion between the outer and inner cylinder. The effective stiffness in this case can be regarded as approximately zero.

In summary, the effective stiffness of the proposed damper is controlled by current $I_{2}$ and it increases as the current $I_{2}$ increases before the current $I_{2}$ reaches a certain high point. In addition, the equivalent damping is mainly controlled by current $I_{1}$. The bigger $I_{1}$ is, the bigger the overall damping. The reasons will be analyzed in section 3. In this model, $x_{1}$ is the relevant displacement between the inner cylinder (connector) and pedestal, $x_{2}$ is the relevant displacement between the outer cylinder and pedestal.

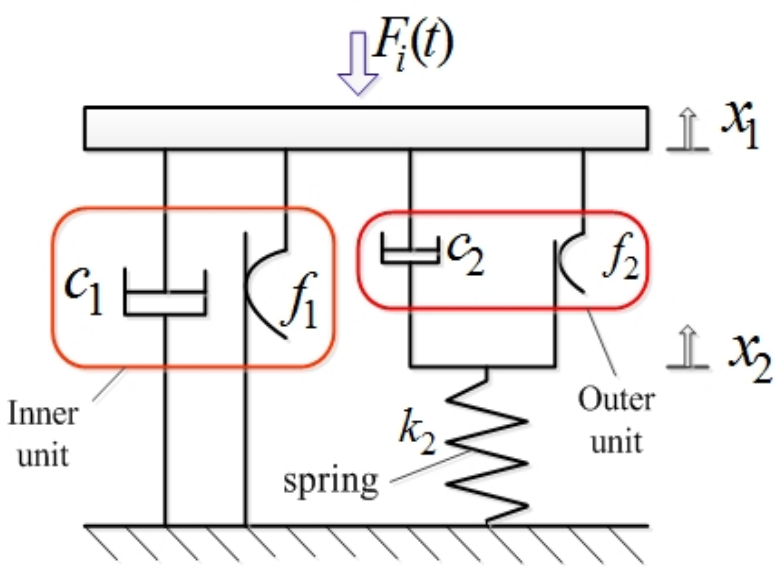

Figure 2. Force model of the new damper

\section{Theoretical analysis}

\subsection{Stiffness analysis}

According to the working principle, the dynamic model of the damper can be equivalent to a variable stiffness spring $k_{v}$ and a variable viscous damping $c_{v}$ which is shown in Figure 3(c). Depending on the current $I_{2}$, the analysis of variable stiffness and damping also scores two situations.

When the current $I_{2}$ is bigger than a certain high point, the spring force is not sufficient to overcome the yield force of MR fluid of outer unit, namely $f_{2}>k_{2} x_{2}$. The viscous element $c_{2}$ is blocked and the inner unit can be equivalent to a variable viscous element $c_{e 1}$, so the dynamic model can be simply represented as the Figure 3(a). Comparing to Figure 3(a) and Figure 3(c), it is easy to obtain that.

$$
\begin{aligned}
& c_{v}=c_{e 1} \\
& k_{v}=k_{2}
\end{aligned}
$$

From Equation (1) and (2), the variable damping of the damper is dependent of $c_{e 1}$ controlled by the current $I_{1}$. While the equivalent stiffness of the damper always is the stiffness of the spring $k_{2}$ which is neither controlled by current $I_{1}$ nor current $I_{2}$.

When the current $I_{2}$ is smaller, there will be a relative motion between the outer and inner cylinder. The viscous element $c_{2}$ is working and the outer unit can be replaced by a variable viscous element $c_{e 2}$, so the model can be simply represented as shown in Figure 3(b). According to Newton's law, a dynamic equation is established.

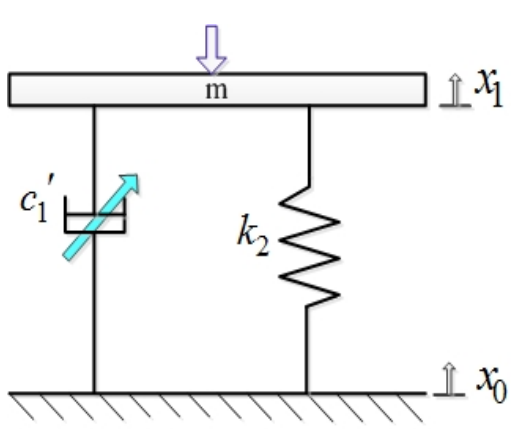

(a) $c_{2}$ blocked

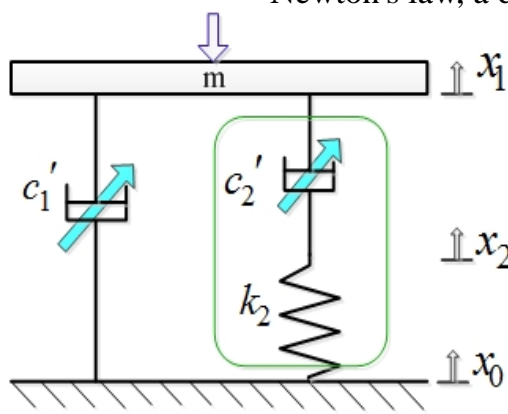

(b) $\mathcal{C}_{2}$ not blocked

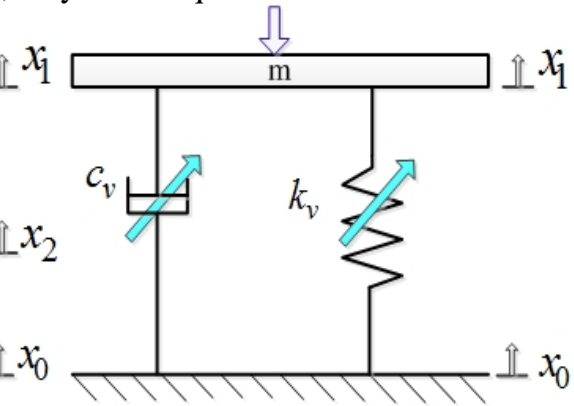

(c) Equivalent

Figure 3. Simplified dynamic model of the new damper 


$$
\begin{aligned}
& m \ddot{x}_{1}=-c_{e 1}\left(\dot{x}_{1}-\dot{x}_{0}\right)-c_{e 2}\left(\dot{x}_{1}-\dot{x}_{2}\right) \\
& c_{e 2}\left(\dot{x}_{1}-\dot{x}_{2}\right)-k_{2}\left(x_{2}-x_{0}\right)=0
\end{aligned}
$$

where $m$ is the counter mass of the damper, $x_{0}$ is the excitation displacement to the damper.

To take the Fourier transform to Equation (3) and (4), a transfer function can be obtained

$$
\frac{X_{1}(j \omega)}{X_{0}(j \omega)}=\frac{k_{2}-k_{2}^{3} /\left(k_{2}^{2}+c_{e 2}{ }^{2} \omega^{2}\right)+\left[c_{e 1}+k_{2}{ }^{2} c_{e 2} /\left(k_{2}^{2}+c_{e 2}{ }^{2} \omega^{2}\right)\right] j \omega}{-m \omega^{2}+k_{2}-k_{2}^{3} /\left(k_{2}{ }^{2}+c_{e 2}{ }^{2} \omega^{2}\right)+\left[c_{e 1}+k_{2}{ }^{2} c_{e 2} /\left(k_{2}^{2}+c_{e 2}{ }^{2} \omega^{2}\right)\right] j \omega}
$$

Similarly, from Figure.3(c), a transfer function also can be obtained.

$$
\frac{X_{1}(j \omega)}{X_{0}(j \omega)}=\frac{c_{v} j \omega+k_{v}}{-m \omega^{2}+c_{v} j \omega+k_{v}}
$$

Comparing to Equation (5) and (6), the equivalent stiffness and damping of the damper can be expressed by

$$
\begin{aligned}
& c_{v}=c_{e 1}+\frac{k_{2}{ }^{2} c_{e 2}}{k_{2}{ }^{2}+c_{e 2}{ }^{2} \omega^{2}} \\
& k_{v}=k_{2}-\frac{k_{2}{ }^{3}}{k_{2}{ }^{2}+c_{e 2}{ }^{2} \omega^{2}}
\end{aligned}
$$

From Equation (7), the variable damping of the damper is mainly dependent of $c_{e 1}$ controlled by the current $I_{1}$ of inner unit when the current $I_{2}$ is small. While from the Equation (8), the equivalent stiffness of the damper is only controlled by $c_{e 2}$ and independent of $c_{e 1}$, that is to say the variable stiffness is only controlled by the current $I_{2}$ of outer unit. It is also seen that the equivalent stiffness is equal to the stiffness of spring $k_{2}$ when the $c_{e 2}$ is infinite. The $c_{e 2}$ is infinite means $c_{2}$ is blocked and the model also can be shown in Figure. 3(a). Meanwhile, the stiffness of the damper is able to zero when the $c_{e 2}$ is equal to zero. But in practice, the minimum $c_{e 2}$ should be equal to the viscosity coefficient $c_{2}$ of the MR fluid when the current $I_{2}$ is zero. From the Equation (1), (2), (7) and (8), the equivalent stiffness $k_{v}$ and damping $c_{v}$ of the new damper can be obtained if the $c_{e 1}$ and $c_{e 2}$ are solved.

\subsection{Damping force analysis}

For the inner unit, the damping force is controlled by the applied current $I_{1}$ and the velocity of MR fluid. No matter what state the outer unit is in, the damping force of inner unit will not be affected. The shear stress and velocity distribution in the ring-working gap are shown in the red dotted box of Figure $4 . u_{1}$ is the relevant velocity between the inner cylinder(connector) and pedestal, $r$ stands for radial direction and $x$ stands for axial direction. The flow direction of MR fluid is the same as motion of the inner cylinder due to the inner piston is stationary, which is different from the work models of other traditional MR dampers, so the damping force needs to be recalculated in theory. The Axisymmetric-model is used here and the shear stress and pressure gradient along the flow direction of the liquid meet the Navier-Stokes equation (Wang et al., 2014).

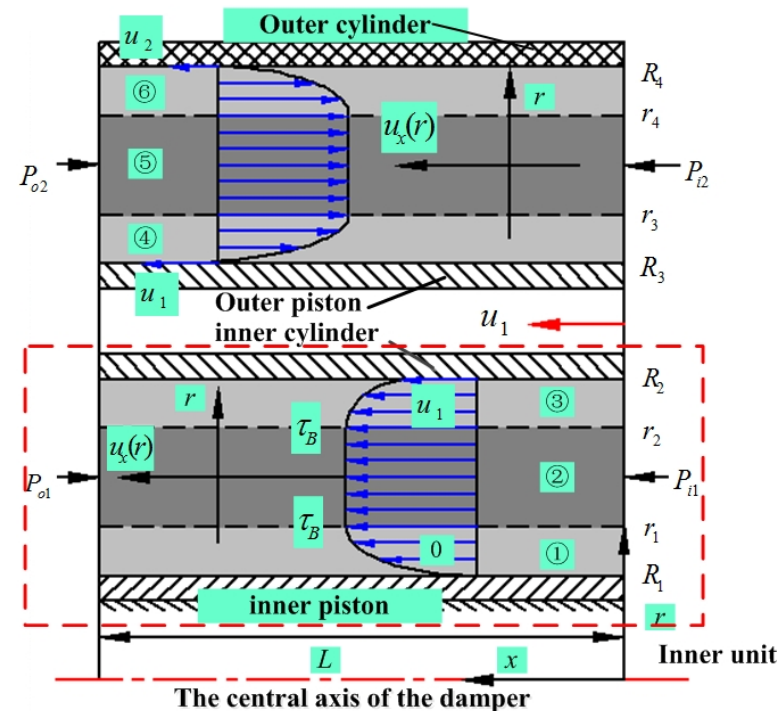

Figure 4. Flow distribution in working gap

$$
\rho \frac{\partial}{\partial t} u_{x}(r)+\frac{\partial}{\partial} \tau_{r x}(r)+\frac{\tau_{r x}}{r}=\frac{\partial p}{\partial x}
$$

where $u_{x}(r)$ is the flow velocity, $\tau_{r x}(r)$ is the shear stress, $\quad p$ is the pressure, $\rho$ is the density of liquid.

The inertial forces of the fluid in the static analysis can be ignored and thus it can be obtained.

$$
\tau_{r x}(r)=\frac{1}{2} \frac{\mathrm{d} p(x)}{\mathrm{d} x} r+\frac{D}{r}
$$

where $D$ is a constant, and it can be solved by boundary conditions.

The damping force is dependent on the crucial properties of the MR fluid, such as the shear yield stress and the no-field viscosity. The constitutive model of the MR fluid can be described by the Bingham plastic model which is expressed as (Li et al., 2000).

$$
\tau_{r x}=\tau_{B}(\operatorname{sgn} \dot{\gamma})+\eta \dot{\gamma}
$$

where $\tau_{B}$ is the shear yield stress of MR fluid in the inner unit depending on the applied magnetic flux density $B, \eta$ is the zero-field viscosity, and $\dot{\gamma}$ is the shear rate.

In the new damper, the parts move in different ways which causes the boundary conditions in the flow field model are different from other traditional dampers. The boundary conditions of are as follows.

The boundary conditions of flow velocity

$$
\begin{aligned}
& u_{x}\left(R_{1}\right)=0 \\
& u_{x}\left(R_{2}\right)=u_{1} \\
& u_{x}\left(r_{1}\right)=u_{x}\left(r_{2}\right)
\end{aligned}
$$

where $R_{1}$ is the outside radius of inner piston, $R_{2}$ is the inner radius of flux sleeve.

The boundary conditions of shear rate

$$
\left.\frac{\mathrm{d} u_{X}(r)}{\mathrm{d} r}\right|_{r=r_{1}}=\left.\frac{\mathrm{d} u_{X}(r)}{\mathrm{d} r}\right|_{r=r_{2}}=0
$$

The boundary conditions of shear stress

$$
\tau_{r x}\left(r_{1}\right)=\tau_{r x}\left(r_{2}\right)=\tau_{B 1}
$$

(16) where $\tau_{B 1}$ is the shear yield stress of MR fluid in the inner unit. 
In the flow area (3), the shear rate $\dot{\gamma}=\mathrm{d} u_{x} / \mathrm{d}_{r} \leq 0$, so the shear stress must be expressed

$$
\tau_{r x}(r)=-\tau_{B 1}+\eta \frac{\mathrm{d} u_{x}}{\mathrm{~d} r}
$$

Substituting equation (10), (11) and boundary condition equations (13), it is obtained that

$$
u_{x}(r)=\int_{r}^{R_{2}} \frac{1}{\eta}\left[\frac{1}{2} \frac{\mathrm{d} p(x)}{\mathrm{d} r} r+\frac{D_{1}}{r}+\tau_{B 1}\right] \mathrm{d} r+u_{1} \quad\left(r_{2} \leq r \leq R_{2}\right)
$$

where $D_{1}$ is a constant, and it can be solved by boundary conditions.

In the flow area (1), the shear rate $\dot{\gamma}=\mathrm{d} u_{x} / \mathrm{d}_{r} \geq 0$, so the shear stress must be expressed

$$
\tau_{r x}(r)=\tau_{B 1}+\eta \frac{\mathrm{d} u_{x}}{\mathrm{~d} r}
$$

Substituting equation (10), (11) and boundary condition equations (12) it is obtained that

$$
u_{x}(r)=\int_{R_{1}}^{r} \frac{1}{\eta}\left[\frac{1}{2} \frac{\mathrm{d} p(x)}{\mathrm{d} r} r+\frac{D_{1}}{r}-\tau_{B 1}\right] \mathrm{d} r \quad\left(R_{1} \leq r \leq r_{1}\right)
$$

In the flow area (1), the boundary condition can be expressed by equations (14). Then combining the equation (18) and (20), it is obtained

$$
\int_{r_{2}}^{R_{2}} \frac{1}{\eta}\left[\frac{1}{2} \frac{\mathrm{d} p(x)}{\mathrm{d} r} r+\frac{D_{1}}{r}+\tau_{B 1}\right] \mathrm{d} r-\int_{R_{1}}^{r_{1}} \frac{1}{\eta}\left[\frac{1}{2} \frac{\mathrm{d} p(x)}{\mathrm{d} r} r+\frac{D_{1}}{r}-\tau_{B 1}\right] \mathrm{d} r=u_{1}
$$

From the boundary condition equation (16) of shear stress and equation (10), it is obtained that

$$
D_{1}=\frac{r_{1} r_{2}}{r_{2}+r_{1}} \tau_{B 1}
$$

In the flow area (2), the shear rate $\dot{\gamma}=\mathrm{d} u_{x} / \mathrm{d}_{r}=0$, so the flow velocity must be always expressed

$$
u_{x}(r)=u_{x}\left(r_{1}\right)=\int_{R_{1}}^{r_{1}} \frac{1}{\eta}\left[\frac{1}{2} \frac{\mathrm{d} p(x)}{\mathrm{d} r} r+\frac{D_{1}}{r}-\tau_{B 1}\right] \mathrm{d} r
$$

The expression of volume flow of MR fluid is (Wang Q et al., 2014).

$$
Q=2 \pi \int_{R_{1}}^{R_{2}} r u_{x}(r) \mathrm{d} r
$$

Substituting equation (20), (22) and (23), the equation (24) can be expressed

$$
\begin{aligned}
& Q=u_{1} A_{p 1}=u_{1} \pi R_{1}^{2} \\
& =2 \pi \int_{R_{1}}^{r_{1}} r u_{x}(r) \mathrm{d} r+2 \pi \int_{r_{1}}^{r_{2}} r u_{x}(r) \mathrm{d} r+2 \pi \int_{r_{2}}^{R_{2}} r u_{x}(r) \mathrm{d} r \\
& =2 \pi \int_{R_{1}}^{r_{1}} r\left\{\int_{R_{1}}^{r} \frac{1}{\eta}\left[\frac{1}{2} \frac{\mathrm{d} p(x)}{\mathrm{d} x}+\frac{D_{1}}{r}-\tau_{B 1}\right] \mathrm{d} r\right\} \mathrm{d} r \\
& +2 \pi \int_{r_{1}}^{r_{2}} r\left\{\int_{R_{1}}^{r_{1}} \frac{1}{\eta}\left[\frac{1}{2} \frac{\mathrm{d} p(x)}{\mathrm{d} x}+\frac{D_{1}}{r}-\tau_{B 1}\right] \mathrm{d} r\right\} \mathrm{d} r \\
& +2 \pi \int_{r_{2}}^{R_{2}} r\left\{\int_{r}^{R_{2}} \frac{1}{\eta}\left[\frac{1}{2} \frac{\mathrm{d} p(x)}{\mathrm{d} x}+\frac{D_{1}}{r}+\tau_{B 1}\right] \mathrm{d} r\right\} \mathrm{d} r
\end{aligned}
$$

where $A_{P 1}$ is the area of inner piston.

From the boundary condition equation (15) of shear rate, it is obtained that

$$
\frac{1}{2} \frac{\mathrm{d} p(x)}{\mathrm{d} x} r_{2}+\frac{D_{1}}{r_{2}}+\tau_{B 1}=\frac{1}{2} \frac{\mathrm{d} p(x)}{\mathrm{d} x} r_{1}+\frac{D_{1}}{r_{1}}-\tau_{B 1}=0
$$

Substituting Equation (22), the Equation (26) can be also expressed

$$
\frac{\mathrm{d} p(x)}{\mathrm{d} x}\left(r_{2}+r_{1}\right)+2 \tau_{B 1}=0
$$

From equation (23),(27)and (29), $\mathrm{dp}(x) / \mathrm{d} x, r_{1}$ and $r_{2}$ can be solved, so the damping force of inner unit is obtained that

$$
F_{d 1}=\Delta p_{1} A_{p 1}=L_{1} \frac{\mathrm{d} p(x)}{\mathrm{d} x} A_{p 1}
$$

where $L_{1}$ is the length of the effective working area of MR fluid in the inner unit, $\Delta p_{1}$ is pressure difference at both ends of the effective working area.

If $f_{2}$ is bigger than the force generated by the spring, there is no relative movement between the outer cylinder and outer piston, the MR fluid in outer unit has not reached the yield stress and doesn't flow, so the inner unit and outer unit can be seen as a whole with compressing the spring. The overall damping $F_{d}$ can be calculated by.

$$
F_{d}=F_{d 1}+k_{2} x_{1}
$$

If $f_{2}$ is smaller than the force generated by the spring, there is relative movement between the outer cylinder and outer piston. The MR fluid in outer unit also should flow, so the shear stress and velocity distribution in the ring gap of the whole damper are shown in the Figure 4.

For the outer unit, the boundary conditions in this model are also different from other traditional dampers, which are as follows.

The boundary conditions of flow velocity are

$$
\begin{aligned}
& u_{x}\left(R_{3}\right)=u_{1} \\
& u_{x}\left(R_{4}\right)=u_{2} \\
& u_{x}\left(r_{3}\right)=u_{x}\left(r_{4}\right)
\end{aligned}
$$

where $u_{2}$ is the relevant velocity between the outer cylinder and pedestal, $R_{3}$ is the outside radius of outer piston, $R_{4}$ is the inside radius of outer cylinder.

The boundary conditions of shear rate

$$
\left.\frac{\mathrm{d} u_{x}(r)}{\mathrm{d} r}\right|_{r=r_{3}}=\left.\frac{\mathrm{d} u_{x}(r)}{\mathrm{d} r}\right|_{r=r_{4}}=0
$$

The boundary conditions of shear stress

$$
\tau_{r x}\left(r_{3}\right)=\tau_{r x}\left(r_{4}\right)=\tau_{B 2}
$$

where $\tau_{B 2}$ is the shear yield stress of MR fluid in the outer unit.

Using a similar solution, the damping force of outer unit can also be obtained

$$
F_{d 2}=\Delta p_{2} A_{p 2}=L_{2} \frac{\mathrm{d} p(x)}{\mathrm{d} x} A_{p 2}
$$

where $L_{2}$ is the length of the effective working area of MR fluid in the outer unit, $\Delta p_{2}$ is pressure difference at both ends of the effective working area .

In this case, the overall damping $F_{d}$ can be calculated by

$$
F_{d}=F_{d 1}+F_{d 2}
$$

The energy dissipated by each unit in one cycle $U_{d i}$ can be given. 


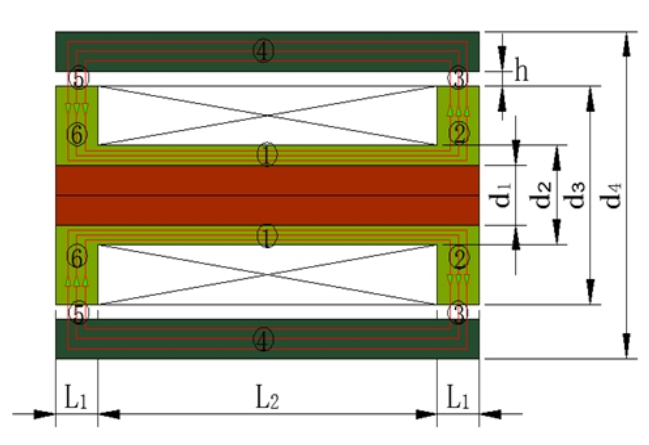

(a) Magnetic circuit and parameters

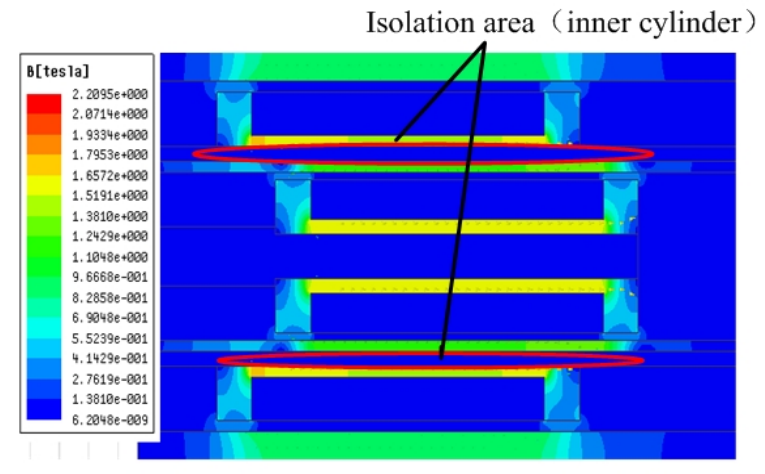

(b) Magnetic circuit(FEA)

Figure 5. Magnetic circuit of the two pistons

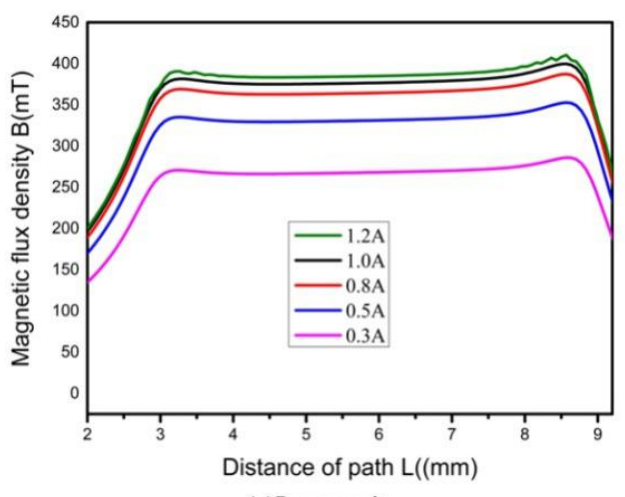

(a)Inner unit

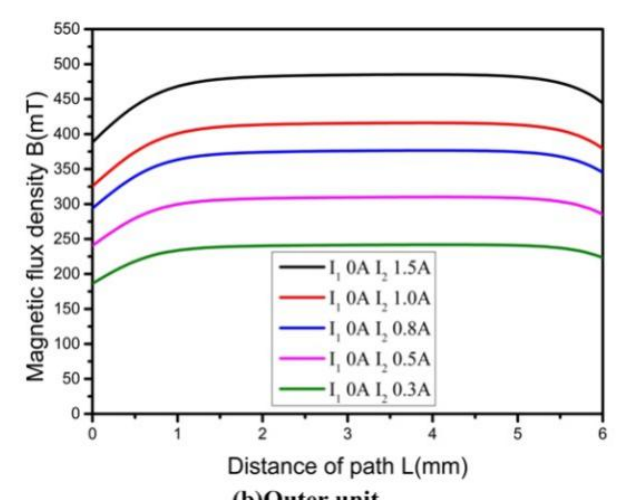

(b)Outer unit

Figure 6. Magnetic flux density in the working gap of MR fluid

$$
U_{d i}=\int F_{d i} \mathrm{~d} x
$$

where $F_{d i}$ is the damping force of each unit, and $i=1$ or 2 . And it is seen that the $U_{d i}$ is the area of hysteresis loop between force and displacement. let

Assume each viscous unit is under sinusoidal excitation,

$$
x_{i}=X_{i} \sin \omega t
$$

where $X_{i}$ is the displacement amplitude, $\omega$ is frequency.

Then the energy dissipated can be expressed by

$$
U_{d i}=\int_{0}^{\frac{2 \pi}{\omega}} F_{d i} \dot{x}_{i} \mathrm{~d} t
$$

While it is known that ( $\mathrm{Li}$ et al. 2000)

$$
U_{d i}=\int c_{e i} \dot{x}_{i} \mathrm{~d} x_{i}
$$

So the equivalent viscous $c_{e i}$ of each unit can be obtained

$$
c_{e i}=\frac{U_{d i}}{\pi \omega X_{i}^{2}}
$$

Substituting Equation (1), (2), (7) and (8), the equivalent stiffness $k_{v}$ and damping $c_{v}$ of the new damper can be obtained.

\subsection{Magnetic field analysis}

The magnetic fields generated by the coils are important for inducing changes in the yield stress of the MR fluid. Figure 5(a) shows the magnetic circuit for both of the two pistons because their structures are the same
Table 1. Main parameters of the prototype.

\begin{tabular}{ccc}
\hline Parameters & Value(inner unit) & Value(outer unit) \\
\hline$d_{1}$ & $6 \mathrm{~mm}$ & $30 \mathrm{~mm}$ \\
$d_{2}$ & $10 \mathrm{~mm}$ & $33 \mathrm{~mm}$ \\
$d_{3}$ & $21 \mathrm{~mm}$ & $45 \mathrm{~mm}$ \\
$d_{4}$ & $26 \mathrm{~mm}$ & $56 \mathrm{~mm}$ \\
$h$ & $1 \mathrm{~mm}$ & $1.5 \mathrm{~mm}$ \\
$L_{1}$ & $6 \mathrm{~mm}$ & $6 \mathrm{~mm}$ \\
$L_{2}$ & $50 \mathrm{~mm}$ & $50 \mathrm{~mm}$ \\
$N_{\text {(turns) }}$ & 500 & 500 \\
\hline
\end{tabular}

and the detailed parameters for both of the two damping units are shown in Table 1.

To verify the magnetic circuit design and get the accurate value of the magnetic flux density applied on the work region, it is necessary to perform finite element analysis (FEA) to analyze the magnetic field of the damper. The inner cylinder is made of stainless steel which is a kind of nonmagnetic material. Thus there is an isolation area to prevent the mutual interference of the magnetic field of inner and outer unit, as shown in Figure 5(b).

To obtain the values of the magnetic flux density in the working gap of MR fluid, the results of the magnetic flux density along each observed path of inner and outer unit are shown in Figure 6 . The applied currents $I_{1}$ for the inner unit is set at $0.3 \mathrm{~A}, 0.5 \mathrm{~A}, 0.8 \mathrm{~A}, 1 \mathrm{~A}$ and $1.2 \mathrm{~A}, I_{2}$ is set at $0 A$, the max magnetic flux density in the work 
region is about $0.36 \mathrm{~T}$. While the applied currents $I_{2}$ for the outer unit is set at $0.3 \mathrm{~A}, 0.5 \mathrm{~A}, 0.8 \mathrm{~A}, 1.0 \mathrm{~A}$ and $1.5 \mathrm{~A}, \mathrm{I}_{1}$ is set at $0 \mathrm{~A}$, the max flux density is about $0.484 \mathrm{~T}$.

\section{Experimental setup}

\subsection{MR fluid selection}

As we know, the most important parameters of the MR fluid are the shear stress, the no-field viscosity. In this study, the MR fluid GH-MRF-45 prepared by Beijing Hao Hua technology limited company is selected. All the performances are tested by Anton Paar MCR 302 rheometer. It is observed that the shear yield stress of the MR fluid under different the magnetic flux density as shown in Figure 7. By fitting the $\tau$-B curve, the relationship between the shear yield stress and the applied magnetic flux density can be expressed as

$$
\tau_{B}=-31.86 B^{4}-41.99 B^{3}+143.8 B^{2}+25.8 B-0.008
$$

\subsection{Prototype of new damper}

Based on the above analysis, a MR fluid damper prototype has been manufactured and assembled as shown in Figure 8. The materials of the prototype components are selected based on Tables 2 .

Table 2 shows the main components and the materials used in the new damper prototype. To avoid the magnetic circuit flowing from the inner unit to the outer one, the inner cylinder and piston rod must be made of nonmagnetic materials. Meanwhile, they are force piece, stainless steel which is a kind of nonmagnetic material with a high mechanical strength are selected. To reduce the magnetic reluctance of the magnetic circuit, the inner piston, outer piston, flux sleeve and outer cylinder are made of DT4A pure iron which has a high magnetic conductivity. The stiffness of the selected spring is $35.8 \mathrm{kN} / \mathrm{m}$.

\subsection{Test of dynamic performance}

As shown in Figure 9(a), a computer-controlled MTS machine was used to test the dynamic performances of the damper. The MTS machine has upper and lower heads with grippers that can hold the damper in place. The lower head is fixed to the bottom base, and the upper head which is excited by the hydraulic cylinder can move up and down at different speeds. A load cell is mounted below the lower head to measure the force generated by the damper. The signals including displacement of inner cylinder and force are saved the computer 1 . In order to test the displacement and speed of outer cylinder, a laser sensor and an aluminium sheet should be installed; the signals are by a data acquisition (DAQ) board (NI USB-6009) and then saved to the computer 2. During the experiment, a DC power is used to generate current to the coils. Once the damper is mounted on the MTS machine using two ends connectors, a predefined routine was programmed into the control software in order to maintain consistency in the testing.

The properties of stiffness variability and damping

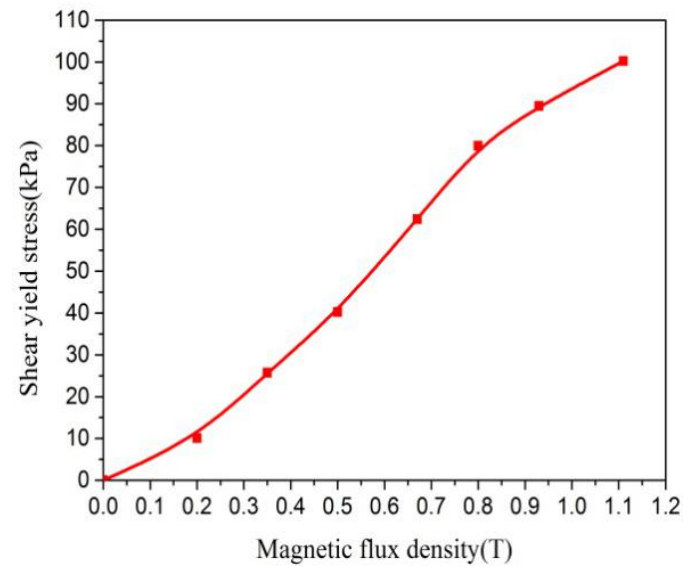

Figure 7. Shear yield stress of MR fluid vs magnetic

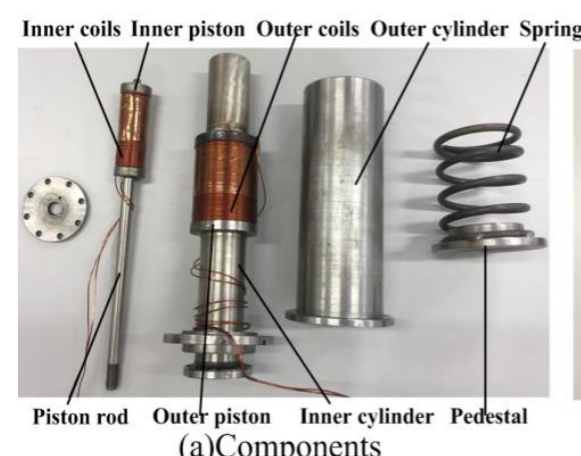

(a)Components

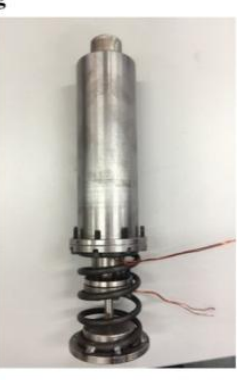

(b)Prototype

Figure 8. Prototype of the new damper

Table 2.Main components of the prototype.

\begin{tabular}{cc}
\hline Component & Material \\
\hline Inner piston & DT4A pure iron \\
Outer piston & DT4A pure iron \\
Piston rod & Stainless steel \\
Inner cylinder & Stainless steel \\
Outer cylinder & DT4A pure iron \\
Flux sleeve & DT4A pure iron \\
\hline
\end{tabular}

variability were first tested separately. To obtain the performance in terms of the variable stiffness of the damper, the excitation signal chosen was a sinusoidal wave with a single frequency of $0.5 \mathrm{~Hz}$ and amplitude of $5 \mathrm{~mm}$, the current $I_{2}$ applied to the outer unit is varied from 0 to $1 \mathrm{~A}$, while the current $I_{1}$ applied to the inner unit is set as $0 \mathrm{~A}$. On the other hand, as for the field-dependent response of variable damping, the signal chosen was a sinusoidal wave with a single frequency of $0.5 \mathrm{~Hz}$ and amplitude of $10 \mathrm{~mm}$, the current $I_{1}$ applied to the inner unit is varied from 0 to $1 \mathrm{~A}$ respectively, with the current $\mathrm{I}_{2}$ maintained as a constant of $0 \mathrm{~A}$. In addition to the fielddependent responses, the effectiveness of changing the loading frequency and amplitude on the damper performances was also investigated. To obtain the frequency-dependent performance, the frequency of predefined sinusoidal routine was changed to $0.5 \mathrm{~Hz}, 1 \mathrm{~Hz}$ and $2 \mathrm{~Hz}$ respectively. For the amplitude-dependent performance, the amplitude was changed to $5 \mathrm{~mm}, 7.5 \mathrm{~mm}$ and $10 \mathrm{~mm}$. For the two testing cases, both the currents $I_{1}$ and $I_{2}$ were set $0 \mathrm{~A}$. 


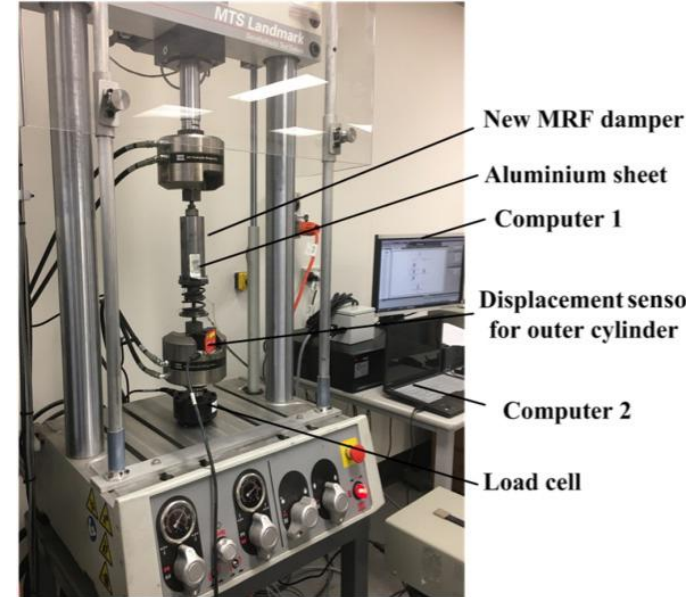

(a)Stiffness and damping test

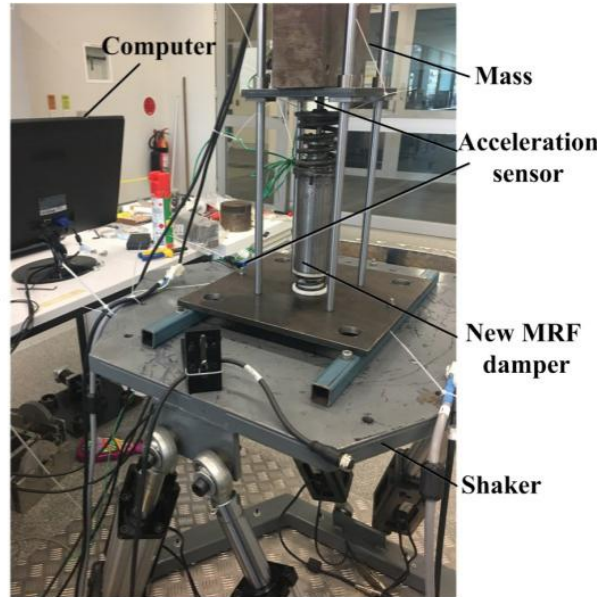

(b)Frequency-shift test

Figure 9. Experiment set-up

For the new damper, the purpose of variable stiffness is to change its natural frequency under the same quality. The frequency-shift property is a key criterion to evaluate the effectiveness and controllability of the new damper. In this experiment, a series of tests using swept sinusoidal signals were conducted to measure the frequency-shift performance. Figure 9(b) illustrates the detailed experimental setup, which mainly consists of the shaker and the horizontal vibration platform. The damper was fixed onto the platform with two accelerometers installed onto the top and bottom plates, respectively, measuring their lateral accelerations. This vibration platform was forced to vibrate horizontally by a shaker driven by a harmonic signal generated by a computer and amplified by a power amplifier. A DC power supply was used to provide current signals to the coils. A data acquisition (DAQ) board was used as the interface between the hardware and the software and transferred the measured accelerations to the computer. The signal collection, recording, and processing were developed using the LabVIEW program. With this system, the transmissibility of the laminated damper with different currents was recorded and displayed directly onto the computer.

\section{Results and discussion}

\subsection{Stiffness testing}

In the experiment of stiffness ability, the current $I_{1}$ is set to $0 \mathrm{~A}$, while the current $I_{2}$ is set to $0 \mathrm{~A}, 0.3 \mathrm{~A}, 0.5 \mathrm{~A}$, $0.8 \mathrm{~A}$ and $1 \mathrm{~A}$ respectively. The force-displacement loops are shown in Figure 10. In order to explain these closed curves more clearly, the case of the current $I_{1}=0 \mathrm{~A}$ and $I_{2}$ $=0.3 \mathrm{~A}$ is taken as an example and the result is shown with a red line in Figure 10. It is seen that the loop is a piecewise function and it can be divided into 6 segments after the inner cylinder starts to move at the displacement of $+5 \mathrm{~mm}$. The segment $I$ is a vertical line, because the motion of the inner cylinder needs to completely overcome the friction inside of the damper and the initial force produced by MR fluid in the inner unit the , and the displacement at the beginning is very small, which means the force is mutated. At segment II, the inner cylinder (connector) keeps to moving. But in this case, the compression of spring is small and the MR fluid in outer unit produces a certain yield stress, so that the elastic force produced is less than the yield force produced by MR fluid in outer unit. There is no relative displacement between the inner and outer cylinder and the viscous element $c_{2}$ is blocked, the out cylinder moves with the inner cylinder and compresses the spring. The spring starts to work, so the segment II is a straight diagonal line and its slope is equal to the stiffness $K_{2}$ of spring. In this segment, the yield stress of MR fluid increases as the current increases, which causes the spring has to be more compressed to overcome the yield force .The segment II becomes longer and all of these segments are parallel, which can be seen from the blue line and red line in Figure 10. At segment III, the spring continues to be compressed to a certain value by the motion of outer cylinder, and its elastic force is equal to by the damping force of the outer unit. In this case, the spring can't be compressed more and remain approximately motionless even though the inner cylinder keeps moving. There must be relative movement between the inner and outer cylinders, so the overall output force is the viscous force and the yield stress MR fluid. When the current $I_{2}$ is a certain value, the yield stress of MR fluid must be constant and the viscous force varies very little with velocity in MR fluid damper ,so the output force keeps constant and the segment III is a horizontal line. The equivalent stiffness of the damper can be calculated by equation (43), which is the slope of the dotted line $\mathrm{AB}$ as shown in Figure 10. The equivalent stiffness can be varied and controlled by the current $I_{2}$ in a certain range.

$$
k_{e q}=\frac{F_{\text {max }}-F_{\text {min }}}{D_{\text {max }}-D_{\text {min }}}
$$

When the current $I_{2}$ is very big, such as $0.8 \mathrm{~A}$, the elastic force always cannot overcome the yield stress of the MR fluid during the movement of inner cylinder, even though the spring is compressed to the 


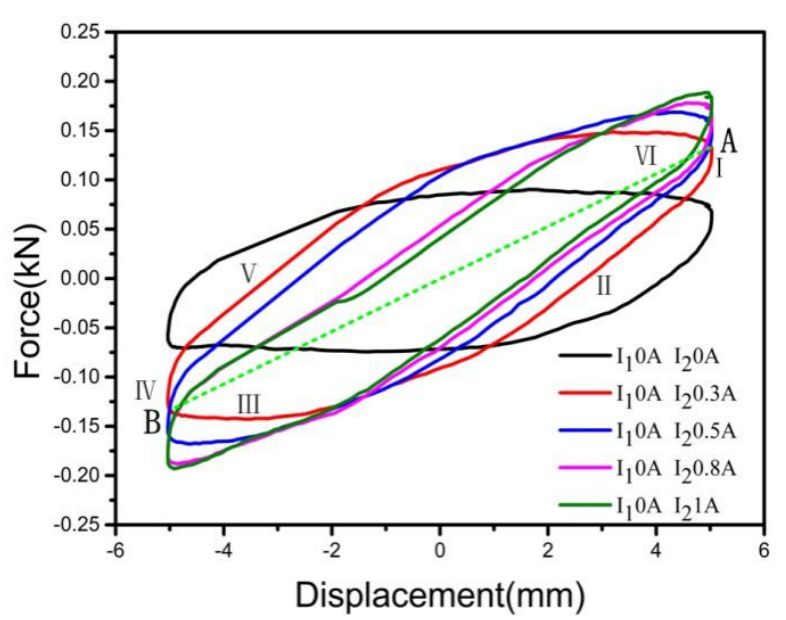

Figure 10. Variable stiffness vs current

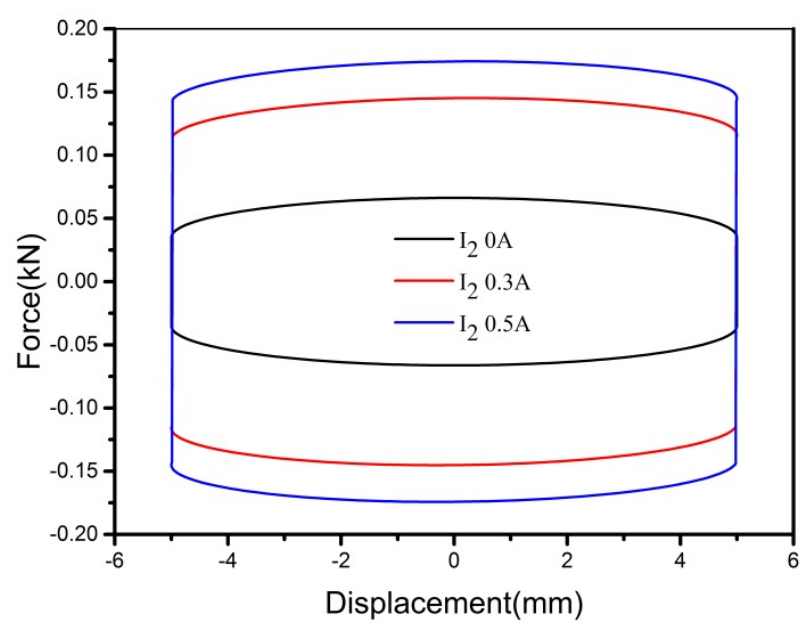

Figure 12. Theoretical damping of outer unit

maximum .In this case ,the spring always works ,so the segment II is longest and the segment III disappears. Spring has been in a working state, so the stiffness of the damper is always equal to the stiffness of the spring, even though the current $I_{2}$ continues to increase. From Figure 10 , the curves almost coincide when the current $I_{2}=0.8 \mathrm{~A}$ and $I_{2}=1 \mathrm{~A}$. It is obtained that and the minimum stiffness of the damper is $13.8 \mathrm{KN} / \mathrm{m}$ and the maximum is $35.8 \mathrm{KN} / \mathrm{m}$ as shown in Figure. 1 . The relative change of stiffness is about $259 \%$, demonstrating that this damper has a wide effective frequency bandwidth. When the inner cylinder reaches the position of $-5 \mathrm{~mm}$, it starts to move back. The process is reversible, and the segments IV, $\mathrm{V}$ and VI are parallel to the segment I, II and III. Selecting the current $I_{2}$ to be 0A.0.5 A and 1A, the forcedisplacement loops obtained by theoretical calculation and experiment is compared, and the results are shown in Figure 11 . When the current $I_{2}$ is $0 \mathrm{~A}$ and $0.5 \mathrm{~A}$, the theoretical values should be calculated by the equation (29) at the segments II and IV, and calculated by the equation (36) at the other segments. When the current $I_{2}$ is $1 \mathrm{~A}$ or $0.8 \mathrm{~A}$, the theoretical values should be calculated by the equation (29) at all segments. In the theoretical calculation of the equivalent stiffness, there are two situations. When the current $I_{2}$ is larger, the equivalent stiffness should be

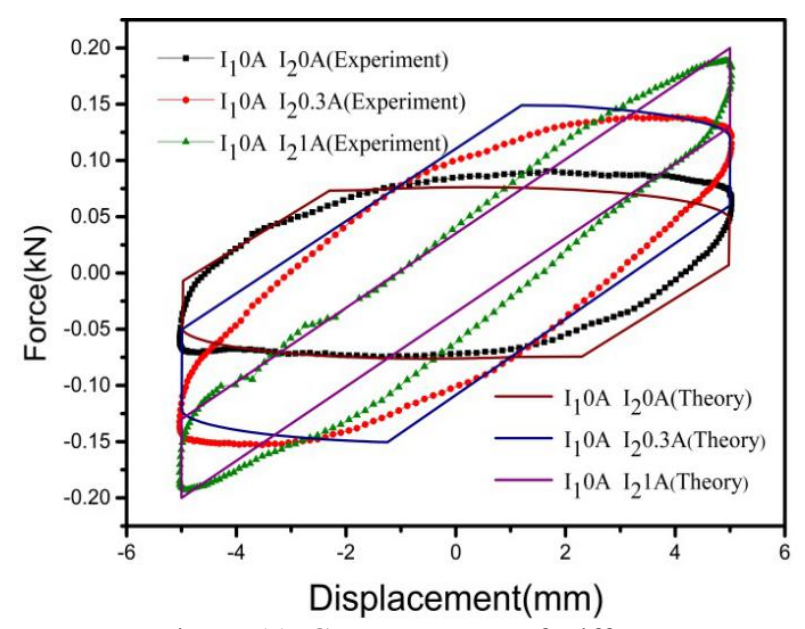

Figure 11. Contrast curve of stiffness

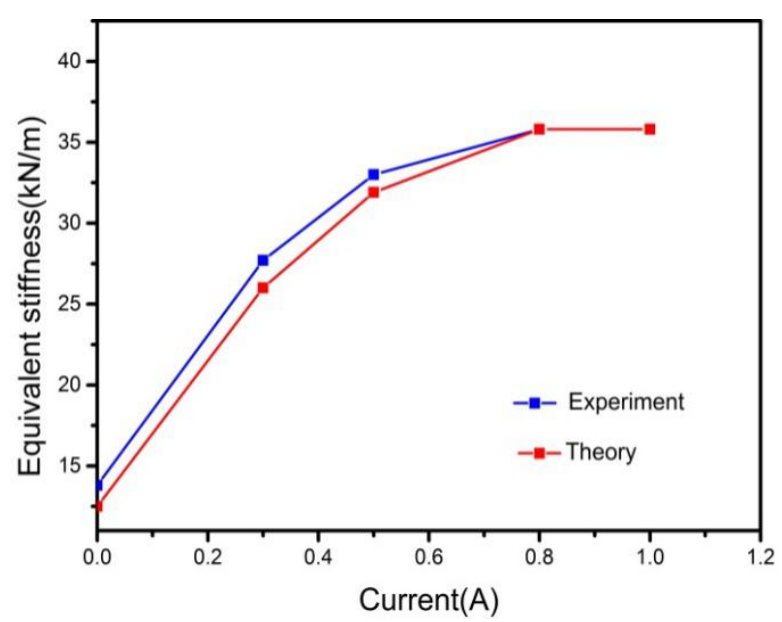

Figure 13. Equivalent stiffness of the damper

calculated by the equation (2), which is the stiffness of spring. When the current is smaller, the equivalent stiffness should be calculated by the equation (8), which has to do with the damping coefficient of outer unit, so the force-displacement loops under different currents must be obtained firstly, and the results are shown in Figure 12. After calculating the damping coefficient of outer unit according to the equation (41), the equivalent stiffness in theory can be obtained as also shown in Figure 13. It is seen that the theoretical stiffness is slightly smaller than the experimental value when the current is less than 0.8A from Figure 13, because some friction inside the damper is ignored in theoretical calculation, which leads to the force is slightly smaller than the experimental value.

\subsection{Damping testing}

From Figure 6 (a), it can be seen that the magnetic flux density at the current $I_{1}$ of $1 \mathrm{~A}$ is almost equal to that at the current $I_{1}$ of $1.2 \mathrm{~A}$, which means the magnetic field starts to saturate at the current $I_{1}$ of $1 \mathrm{~A}$, so the max current was chosen as $1 \mathrm{~A}$ in both experiment and theoretical calculation. Under different currents of $I_{1}=0 \mathrm{~A}, 0.5 \mathrm{~A}$ and $1 \mathrm{~A}, I_{2}=0 \mathrm{~A}$, the variable damping characteristic is shown in Figure 14, it can be seen that the area of enclosed curve of force-displacement increases as the current increases, 


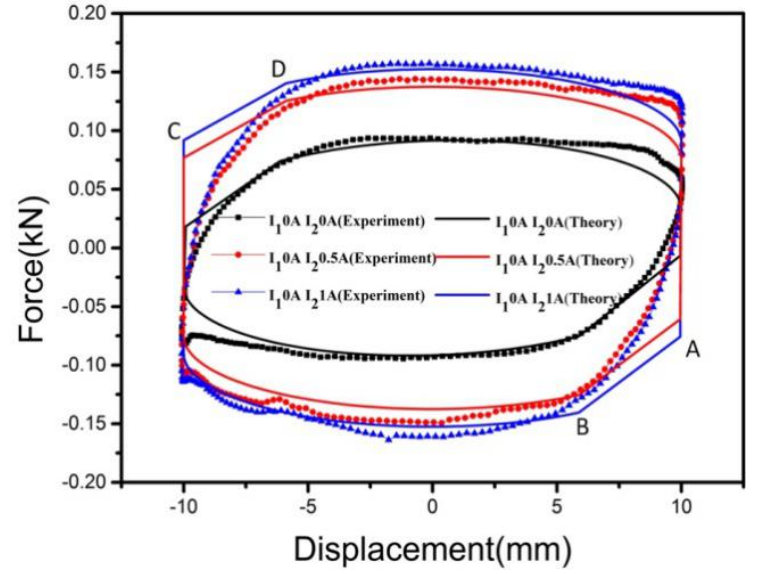

Figure 14. Variable damping vs current

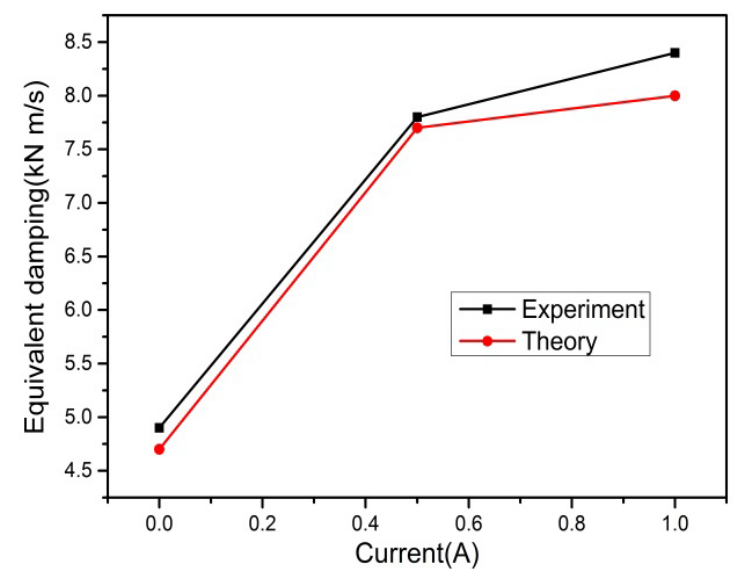

Figure 16. Equivalent damping vs current

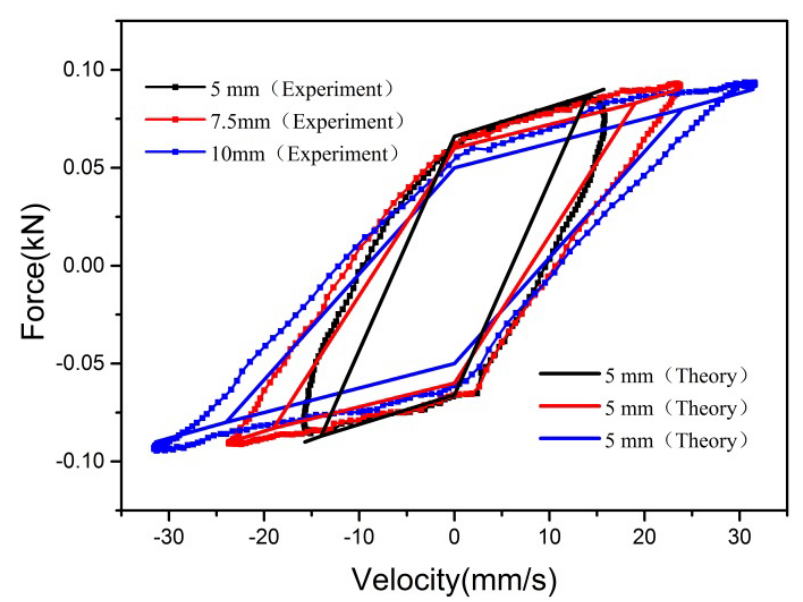

Figure 18. Force- velocity characteristic vs amplitudes

which means the increase of current $I_{1}$ will result in an increase in the equivalent damping coefficient of the damper. This result can be also obtained from the Figure 15 of force- velocity characteristic. The maximum output force obtained by theoretical calculation is slightly smaller than that of the experiment because of some friction in the actual damper, which leads to the equivalent damping coefficient obtained by theoretical calculation is also slightly smaller than that of the experiment, as shown in Figure 16. The minimum damping coefficient is 4.9 $\mathrm{KN}\left(\mathrm{m} \mathrm{s}^{-1}\right)^{-1}$ obtained by

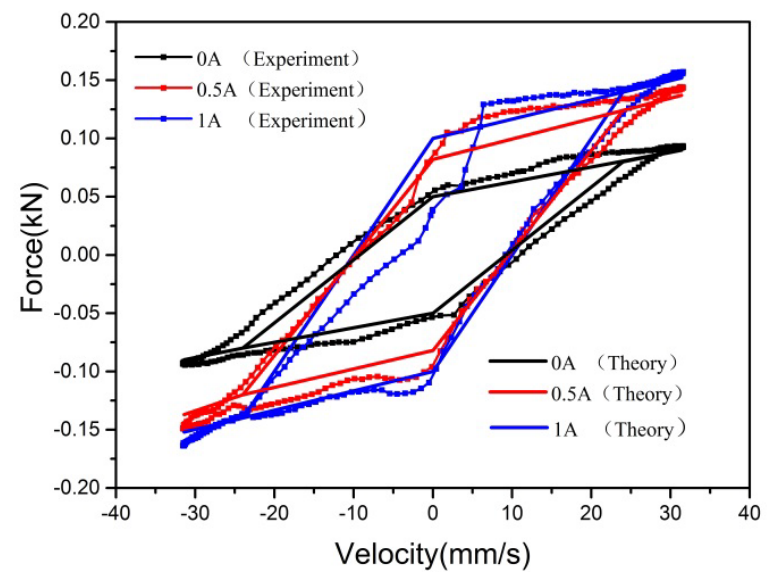

Figure 15. Force- velocity characteristic vs current

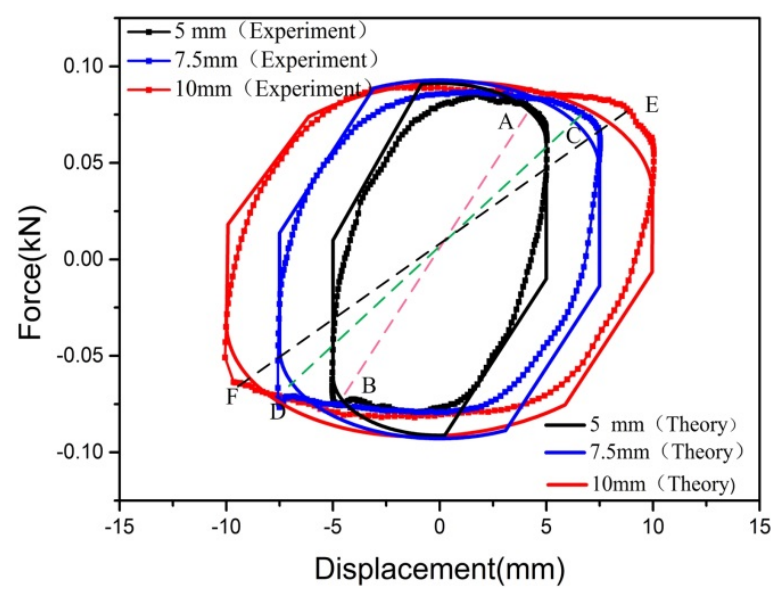

Figure 17. Different amplitudes

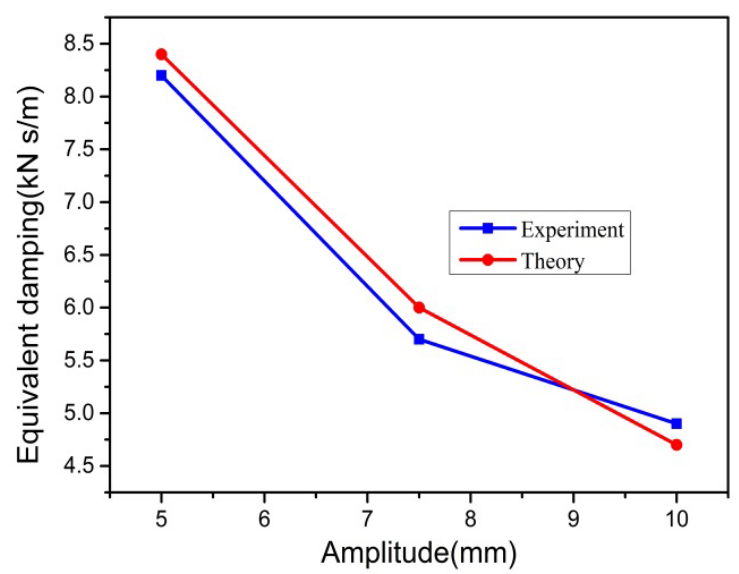

Figure 19. Equivalent damping vs amplitude

equation (41) and the maximum is $8.4 \mathrm{KN}(\mathrm{m} \mathrm{s}-1)-1$. As a result of the zero-field viscous force of MR fluid and friction between the inner and outer cylinders, the outer cylinder drives the spring to work in a small displacement, which is indicated by the segment $\mathrm{AB}$ in Figure 14 . Likewise, the segment CD appears when the movement returns.

\subsection{Responses of amplitude and frequency}

In the case of the current $I_{1}=0 \mathrm{~A}$ and $I_{2}=0 \mathrm{~A}$, the experiments are done to test the effects on the output of 


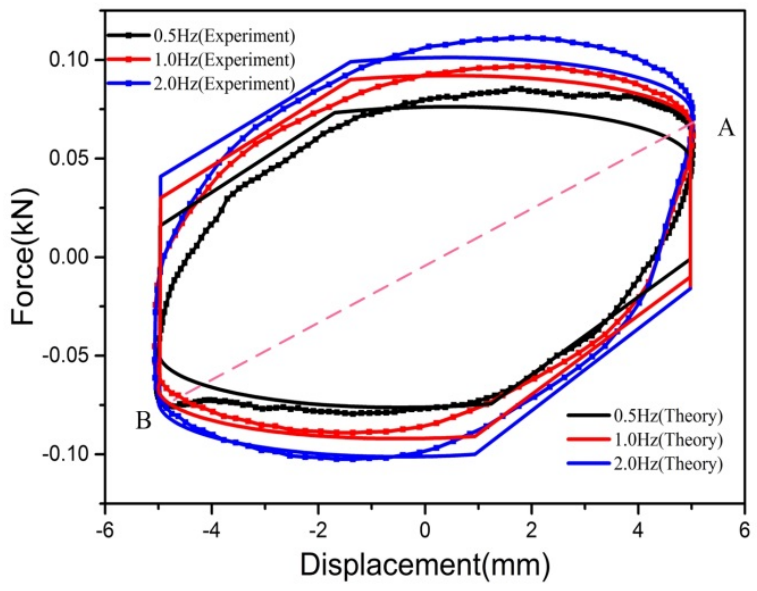

Figure 20. Different frequencies

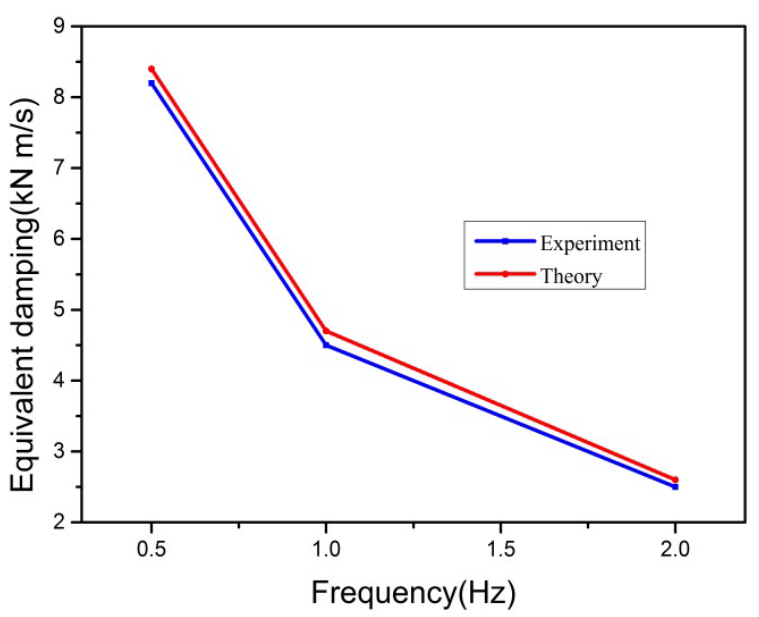

Figure 22. Equivalent damping vs frequency

the MRF damper by changing the frequency and amplitude of sinusoidal motion respectively. Figure 17 shows the influences on the output of damper under different amplitude $5 \mathrm{~mm}, 7.5 \mathrm{~mm}$ and $10 \mathrm{~mm}$ respectively while the frequency is set to $0.5 \mathrm{~Hz}$. It can be seen the maximum output forces are almost the same, but the equivalent damping coefficients are greatly affected which can be calculated by equation (41). The equivalent damping coefficient decreases as the amplitude increases which can also be reflected from the slopes in the Figure 18 of Force- velocity characteristic under different amplitudes and the final result is shown in the Figure 19. The equivalent stiffness also decreases slightly as the amplitude increases which can be obtained by the slopes of the dotted lines $\mathrm{AB}, \mathrm{CD}$ and $\mathrm{EF}$ in Figure 17. Figure 20 and 21 show the responses of the damper under different frequency $0.5 \mathrm{~Hz}, 1 \mathrm{~Hz}$ and $2 \mathrm{~Hz}$ respectively. The amplitude is set to $5 \mathrm{~mm}$. It can be seen that the area of closed curve increases slightly as the frequency increases, but the equivalent-damping coefficient decreases as the frequency increases which can be calculated by equation (41) and the final result is shown in the Figure 22. From the dotted line $A B$ in Figure 20, it is also seen that the frequency variation has little effect on the equivalent stiffness.

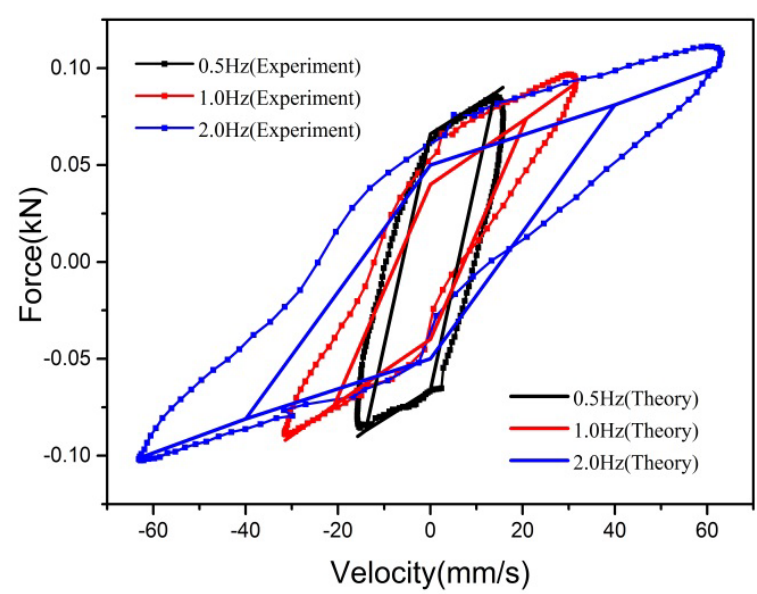

Figure 21. Force- velocity characteristic vs frequencies

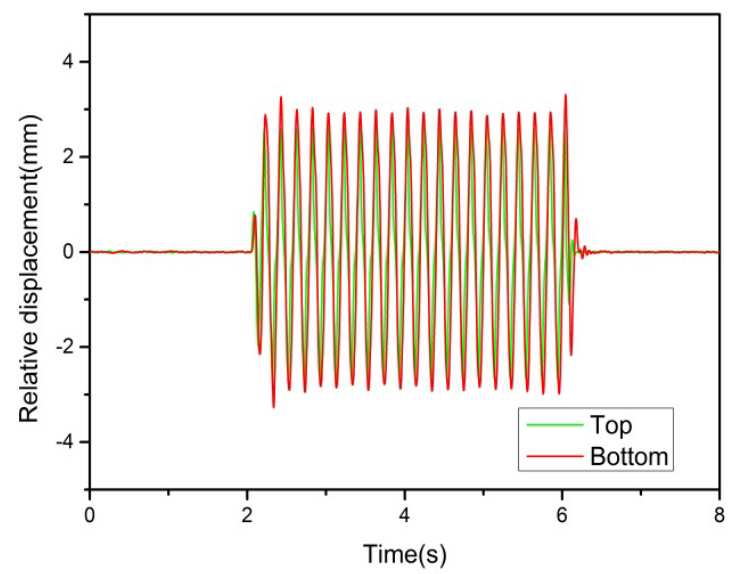

Figure 23. Relative displacement between top and bottom

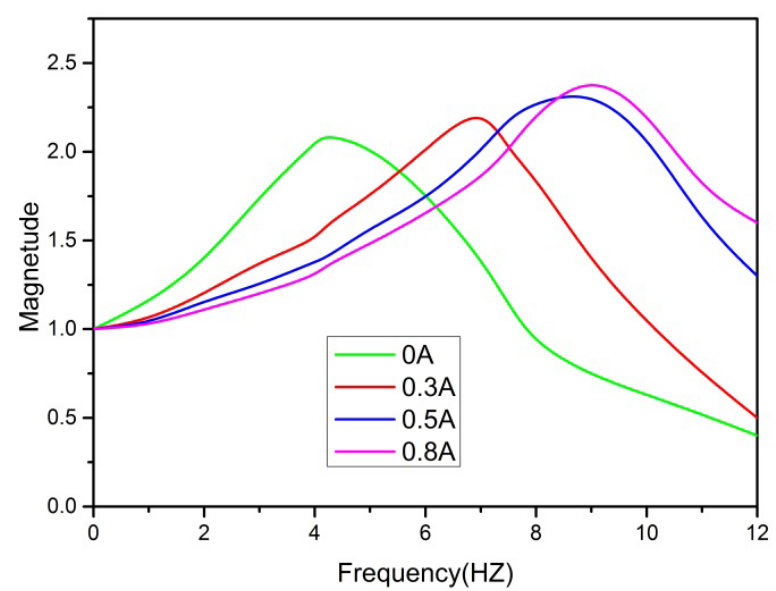

Figure 24. Transmissibility of the damper

\subsection{Frequency-shift}

In this test, the DC current for outer unit was set at $0 \mathrm{~A}$, $0.3 \mathrm{~A}, 0.5 \mathrm{~A}$ and $0.8 \mathrm{~A}$. The resonance principle is used to test the natural frequency of the new damper. When the displacement of bottom is consistent with that of top as shown in Figure 23, the frequency of excitation is equal to the natural frequency of the damper. Figure 24 records the frequency-shift performances (transmissibility) under 
various current conditions. It is seen that the natural frequency increased from $4.1 \mathrm{~Hz}$ to $9.6 \mathrm{~Hz}$. The relative change of the natural frequency was about 235\%, which is close to the relative change of stiffness.

\section{Conclusion}

In this study, a new compact variable damping and stiffness MR Fluid damper is designed, developed, modeled and tested. The results from both calculation in theory and test verified that the damping and stiffness properties can be controlled by current respectively. Due to the boundary conditions in the flow area of the new damper are different from other traditional dampers, the damping force has been calculated in theory and compared with the experimental results in this paper, which illustrates the rationality of the structure and the correctness of the model. From the results, the stiffness of the damper can vary from $13.8 \mathrm{kN} / \mathrm{m}$ to $35.8 \mathrm{kN} / \mathrm{m}$ when the current applied to the outer unit is increased from $0 \mathrm{~A}$ to $1 \mathrm{~A}$ and the max stiffness is also related to the selected spring . The equivalent damping coefficient can vary from $4.9 \mathrm{kN}\left(\mathrm{m} \mathrm{s}^{-1}\right)^{-1}$ to $8.4 \mathrm{kN}\left(\mathrm{m} \mathrm{s}^{-1}\right)^{-1}$. It should be noted that the stiffness can be determined by current $I_{2}$ firstly and then the damping can be determined by current $I_{1}$ in practical application. The successful development variable stiffness and damping proves the concept of variable stiffness and damping.

\section{Acknowledgements}

This work was supported by the Key Laboratory of Fluid Power and Intelligent Electro-Hydraulic Control (Fuzhou University), Fujian Province University, the Science and Technology Major Project of Fujian Province (grant number 2011HZ006-1), Construction of Scientific and Technological Innovation Platform of Fujian Province (grant number 2011H2008), and Special Funds for the University Development from Central Finance of China in 2012 and 2016.

\section{References}

Behrooz M, Wang X and Gordaninejad F (2014) Modeling of a new semi-active/passive magnetorheological elastomer isolator. Smart Materials and Structures 23 (4):045013

Choi YT, Wereley NM and Jeon YS (2005) Semi-active vibration isolation using magnetorheological isolators. Spie Smart Structure \& Materials Symposium 42: 1244-1251.

Deng HX, Gong XL and Wang LH (2006) Development of an adaptive tuned vibration absorber with magnetorheological elastomer. Smart Materials and Structures 15(5):111-116

Du H, Sze KY and Lam J (2005) Semi-active control of vehicle suspension with magneto-rheological dampers. Journal of Sound and Vibration 283 (283):981-996.

Greiner P, Tan A and Sattel T (2014) A semi-active magnetorheological fluid mechanism with variable stiffness and damping. Smart Materials and Structures 23(11): 115008.

Gsell D, Feltrin G and Motavalli M (2007) Adaptive tuned mass damper based on pre-stressable leaf-spring. Journal of Intellignet Material Systems and Structures. 18(8):845-851.

Hitchcock GH, Elkins J and Zhang Q et al. (2004) Tunable magneto-rheological elastomers and processes for their manufacture. US Patent Specification 7261834.

Kwangjin L (1997) Numerical modeling for the hydraulic performance prediction of automotive monotube dampers. Vehicle System Dynamics 28(1):25-39.

Li WH, Du H and Guo N (2003) Design and testing of an MR steering damper for motorcycles. International Journal of Advanced Manufacturing Technology 22(3-4):288-294.

Li WH, Wang XY and Zhang XZ et al.(2009) Development and analysis of a variable stiffness damper using an MR bladder. Smart Materials and Structures 18 (7):074007.

Li WH, Yao GZ and Chen G et al. (2000) Testing and steady state modeling of a linear MR damper under sinusoidal loading. Smart Materials and Structures 9(1):95-102.

Li Y, Li J and Tian T et al. (2013) A highly adjustable magnetorheological elastomer base isolator for applications of real-time adaptive control. Smart Materials and Structures 22(9): 095020.

Liu Y, Matsuhisa H and Park JG et al. (2005) Vibration isolation by a variable stiffness and damping system. Jsme International Journal 48(2):16-28.

Liu Y, Matsuhisa H and Utsuno H (2008) Sem-active vibration isolation system with variable stiffness and damping control. Journal of Sound and Vibration 313(1):16-28.

Raja P, Wang J and Gordaninejad (2014) A high-force controllable MR fluid damper-liquid spring suspension system. Smart Materials and Structures 23(1): 015021.

Spencer BF, Sain MK and Carlson JD (1998) An experimental study of MR dampers for seismic protection. Smart Materials and Structures 7(5):693703.

Sun S, Deng HX and Li WH (2014) Variable stiffness and damping suspension system for train. Active and Passive Smart Structures and Integrated Systems SPIE 9057(15):4177-4180.

Sun S, Deng HX and Li WH et al. (2015) A compact variable stiffness and damping shock absorber for vehicle suspension. IEEE/AMSE Transaction on Mechatronics 20 (5): 2621-2629.

Sun S, Yang J and Li WH et al. (2015) Development of a novel variable stiffness and damping magnetorheological fluid damper. Smart Materials and Structures 24 (8):085021.

Sun S, Yang J and Li WH et al. (2017) Development of an isolator working with magnetorheological elastomers and fluids. Mechanical Systems and Signal Processing 83:371-384.

Talbot JP (2004) Base isolated buildings: Modeling for 
the prediction of isolation performance. Acoustics Bulletin 29 (6) 26-31.

Wang Q, Mehdi A and Chen ZB (2014) A novel doublepiston magnetorheological damper for space truss structures vibration suppression. Shock and Vibration 2014 (1) :1-11.

Worden K and Tomlinson GR (1992) Parametric and nonparametric identification of automotive shock absorber. Modal Analysis 10: 57-62.

Youn I and Hac A (1995) Semi-active suspensions with adaptive capability. Journal of Sound and Vibration
180 (3) :475-492.

Zhang XZ, Li WH and Gong XL et al. (2008) An effective permeability model to predict-dependent modulus of magnetorheological elastomers. Communications in Nonlinear Science And Numerical Simulation 13: 1910-1906.

Zhang XZ, Wang XY and Li WH et al. (2009) Variable stiffness and damping MR isolator. Journal of Physics: Conference Series 149 (1) :012088. 